\title{
Eigen Spectra for q-Deformed Hyperbolic Scarf Potential Including a Coulomb-like Tensor Interaction
}

\author{
M. Eshghi ${ }^{1, *}$ and H. Mehraban ${ }^{2}$ \\ ${ }^{1}$ Physics Department, Islamic Azad University, Central Tehran Branch, P.O. Box 13185-768, \\ Tehran, Iran \\ ${ }^{2}$ Physics Department, Semnan University, P.O. Box 35195-363, Semnan, Iran
}

Received 22 March 2011, accepted 7 April 2011

\begin{abstract}
We study the Dirac equation for the q-deformed hyperbolic Scarf potential including a coulomb-like tensor potential under the spin symmetry. The parametric generalization of the Nikiforov-Uvarov method is used to obtain the energy eigenvalues equation and the unnormalized wave functins.
\end{abstract}

Keywords: Dirac equation; q-deformed hyperbolic Scarf; Spin symmetry; Tensor coupling.

(c) 2011 JSR Publications. ISSN: 2070-0237 (Print); 2070-0245 (Online). All rights reserved.

doi:10.3329/jsr.v3i2.7295 J. Sci. Res. 3 (2), 239-247 (2011)

\section{Introduction}

The description of phenomena at high energies requires the investigation of relativistic wave equations. Dirac equation describes the dynamics of spin- $1 / 2$ particle $[1,2]$ has been used in solving many problems of nuclear and high-energy physics. The spin symmetry appears when the magnitude of the scalar and vector potentials are nearly equal, i.e., $V_{v}(r) \cong V_{s}(r)$, in the nuclei while the pseudospin symmetry occurs when $V_{v}(r) \cong-V_{s}(r)$ [3]. The spin symmetry is relevant for mesons [4] and the pseudospin symmetry has been used to explain the features of deformed nuclei [5], superdeformation [6], and to establish an effective nuclear shell-model scheme [7, 8]. The solutions of the Dirac equation having the pseudospin and spin symmetries have been strongly studied in recent years. The Dirac equation under the spin and/or pseudospin symmetry has been investigated by with different type of potentials such as the Mie-type potential [9], Coulomb-like potential [10], Wood-Saxon potential [11], Eckart potential [12], the generalized Poschl-Teller potential [13], the Makarov potential [14], the Davidson potential [15], the special Hulthen potential [16], the Hyperbolic potential [17], etc.

\footnotetext{
*Corresponding author: eshgi54@gmail.com
} 
On the other hand, tensor potentials were introduced into the Dirac equation with the substitution $\vec{p} \rightarrow \vec{p}-i m \omega \beta \cdot \hat{r} U(r)$. In this way, a spin-orbit coupling term is added to the Dirac Hamiltonian [18-25].

The q-deformed hyperbolic Scarf potential is defined by $\alpha r>\ln \sqrt{q}[26,27]$,

$$
V_{q}(r)=V_{0}+V_{1} \operatorname{coth}_{q}^{2} \alpha r+V_{2} \frac{\operatorname{coth}_{q} \alpha r}{\sinh _{q} \alpha r} .
$$

We write this potential that may be used in decribing the nuclei interactins. Recently, Nikiforov-Uvarov method has been introduced for solving the Schrodinger, KlienGordon, Dirac equations. These equations with the well known potentials are solved by using the Nikiforov-Uvarov method [28-44]. This method has shown its power in calculating the exact energy levels for some solvable quantum systems. Our aim in this paper, is to attempt to study the Dirac equation with q-deformed Hyperbolic Scarf potential (1) for s-wave including a coulomb-like tensor potential

$$
U(r)=-\frac{H}{r}, \quad H=\frac{Z_{a} Z_{b} e^{2}}{4 \pi \varepsilon_{0}}, \quad r \geq R_{c} .
$$

(where $R_{c}=7.78 \mathrm{fm}$ is the Coulomb radius, $Z_{a}$ and $Z_{b}$ denote the charges of the projectile $a$ and the target nuclei $b$, respectively.) under the spin symmetry. We obtain the bound state energy equation and the corresponding spinor wave functions by using the parametric generalization of the Nikiforov-Uvarov method [20, 45].

\section{Dirac equation}

According to the report given in the researcher [18-22], the Dirac equation with the scalar potential $V_{s}(r)$, vector potential $V_{v}(r)$ and a tensor potential $U(r)$ reads $(\hbar=c=1)$ :

$$
\left[\alpha \cdot P+\beta\left(M+V_{s}(r)\right)-i \beta \alpha \cdot \hat{r} U(r)\right] \psi_{n k}(\vec{r})=\left[E-V_{v}(r)\right] \psi_{n k}(\vec{r}),
$$

where $E$ is the relativistic energy of the system, $\vec{P}=-i \vec{\nabla}$ is the momentum operator, $\alpha$ and $\beta$ are the $4 \times 4$ matrices. For spherical nuclei, the angular momentum $\vec{J}$, and $\hat{K}=-\beta(\hat{\sigma} \cdot \hat{L}+1)$ commute with the Dirac Hamiltonian, where $L$ is orbital angular momentum. The Dirac spinor can be classified according to their angular momentum $j, k$,

$$
\psi_{n k}(r)=\frac{1}{r}\left[\begin{array}{l}
F_{n k}(r) Y_{j m}^{l}(\theta, \phi) \\
i G_{n k}(r) Y_{j m}^{\tilde{l}}(\theta, \phi)
\end{array}\right], \quad k=\left(j+\frac{1}{2}\right),
$$




$$
\psi_{n k}(r)=\frac{1}{r}\left[\begin{array}{l}
F_{n k}(r) Y_{j m}^{\tau}(\theta, \phi) \\
i G_{n k}(r) Y_{j m}^{l}(\theta, \phi)
\end{array}\right], \quad k=-\left(j+\frac{1}{2}\right),
$$

where $Y_{j m}^{l}(\theta, \phi)$ and $Y_{j m}^{\tilde{l}}(\theta, \phi)$ are the spin and pseudospin spherical harmonics functions, respectively, $F_{n k}(r)$ and $G_{n k}(r)$ are the radial wave functions of the upperand the lower-spinor components respectively, $m$ is the projection of the total angular momentum on the $z$-axis, $n$ is the radial quantum number. The orbital and the pseudoorbital angular momentum quantum numbers for spin symmetry $l$ and pseudo-spin symmetry $\tilde{l}$ refer to the upper- and lower-component respectively.

Substituting (4) and (5) into (3) and using the following relations [2]

$$
\begin{aligned}
& (\vec{\sigma} \cdot \vec{A})(\vec{\sigma} \cdot \vec{B})=\vec{A} \cdot \vec{B}+i \vec{\sigma} \cdot(\vec{A} \times \vec{B}), \\
& (\vec{\sigma} \cdot \vec{P})=\vec{\sigma} \cdot \hat{r}\left(\hat{r} \cdot \vec{P}+i \frac{\vec{\sigma} \cdot \vec{L}}{r}\right),
\end{aligned}
$$

and properties

$$
\begin{aligned}
& (\vec{\sigma} . \vec{L}) Y_{j m}^{\tilde{l}}(\theta, \phi)=(k-1) Y_{j m}^{\tilde{l}}(\theta, \phi), \\
& (\vec{\sigma} \cdot \vec{L}) Y_{j m}^{l}(\theta, \phi)=-(k-1) Y_{j m}^{l}(\theta, \phi), \\
& (\vec{\sigma} . \hat{r}) Y_{j m}^{\tilde{l}}(\theta, \phi)=-Y_{j m}^{l}(\theta, \phi), \\
& (\vec{\sigma} . \hat{r}) Y_{j m}^{l}(\theta, \phi)=-Y_{j m}^{\tilde{l}}(\theta, \phi),
\end{aligned}
$$

Splitting off the angular part and leaving the radial wave function satisfy the following equations

$$
\begin{aligned}
& \left(\frac{d}{d r}+\frac{k}{r}-U(r)\right) F_{n k}(r)=\left[E_{n k}+M-\Delta(r)\right] G_{n k}(r), \\
& \left(\frac{d}{d r}-\frac{k}{r}+U(r)\right) G_{n k}(r)=\left[M-E_{n k}+\Sigma(r)\right] F_{n k}(r),
\end{aligned}
$$

where $\Delta(r)=V_{v}(r)-V_{s}(r)$ and $\Sigma(r)=V_{v}(r)+V_{s}(r)$. To eliminating $G_{n k}(r)$ in (12) and $F_{n k}(r)$ in (13), one obtains Schrodinger-like equations for the upper and lower components, respectively

$$
\left\{\frac{d^{2}}{d r^{2}}-\frac{k(k+1)}{r^{2}}+\frac{2 k}{r} U(r)-\frac{d U(r)}{d r}-U^{2}(r)+\left(E_{n k}+M-\Delta(r)\right)\left(E_{n k}-M-\Sigma(r)\right)\right.
$$




$$
\begin{aligned}
& \left.+\frac{\frac{d \Delta(r)}{d r}}{\left(M+E_{n k}-\Delta(r)\right)}\left(\frac{d}{d r}+\frac{k}{r}-U(r)\right)\right\} F_{n k}(r)=0, \\
& \left\{\frac{d^{2}}{d r^{2}}-\frac{k(k-1)}{r^{2}}+\frac{2 k}{r} U(r)+\frac{d U(r)}{d r}-U^{2}(r)\right. \\
& +\left(E_{n k}+M-\Delta(r)\right)\left(E_{n k}-M-\Sigma(r)\right) \\
& \left.+\frac{d \Sigma(r)}{\left(M-E_{n k}+\Sigma(r)\right)}\left(\frac{d}{d r}-\frac{k}{r}+U(r)\right)\right\} G_{n k}(r)=0,
\end{aligned}
$$

where $k(k+1)=l(l+1)$ and $k(k-1)=\tilde{l}(\tilde{l}+1)$.

We applied deform hyperbolic functions introduced for the first time by Arai [46]

$$
\begin{array}{ll}
\sinh _{q} x=\frac{e^{x}-q e^{-x}}{2}, & \cosh _{q} x=\frac{e^{x}+q e^{-x}}{2}, \\
\tanh _{q} x=\frac{\sinh _{q} x}{\cosh _{q} x}, & \operatorname{sech}_{q} x=\frac{1}{\cosh _{q} x},
\end{array}
$$

where $q$ is real parameter and $q>0$.

\subsection{Spin symmetry for s-wave}

Substituting (1) into (14), $U(r)=0$ and considering spin symmetry (the condition of spin symmetry $d \Delta(r) / d r=0$ or $\Delta(r)=$ const $=C_{s}$ ) [47, 48], we obtain the second-order differential equation for the upper component of the Dirac spinor as follows,

$$
\begin{aligned}
& \left\{\frac{d^{2}}{d r^{2}}+\left(E_{n k}+M-C_{s}\right)\left(E_{n k}-M\right) \cdot\right. \\
& \left.-\left(E_{n k}+M-C_{s}\right)\left(V_{0}+V_{1} \operatorname{coth}_{q}^{2} \alpha r+V_{2} \frac{\operatorname{coth}_{q} \alpha r}{\sinh _{q} \alpha r}\right)\right\} F_{n k}(r)=0,
\end{aligned}
$$

and $s=\cosh _{q} \alpha r$, we have

$\left\{\frac{d^{2}}{d s^{2}}+\frac{-s}{q-s^{2}} \frac{d}{d s}+\frac{1}{\left(q-s^{2}\right)^{2}}\left[-b_{1}\left(b_{2}+\tilde{V}_{0}+\tilde{V}_{1}\right) s^{2}+b \tilde{V}_{2} s+\left(b_{2}+\tilde{V}_{0}\right) b_{1} q\right)\right] F_{n k}(s)=0$,

where

$$
b_{1}=E_{n k}+M-C_{s}, \quad b_{2}=\frac{M-E_{n k}}{\alpha^{2}},
$$




$$
\tilde{V}_{0}=\frac{V_{0}}{\alpha^{2}}, \quad \tilde{V}_{1}=\frac{V_{1}}{\alpha^{2}}, \quad \tilde{V}_{2}=\frac{V_{2}}{\alpha^{2}} .
$$

Following to the report given in the researcher [20,45], we obtain the parameter set as

$$
\begin{aligned}
& \xi_{1}=b_{1}\left(b_{2}+\tilde{V}_{0}+\tilde{V}_{1}\right), \quad \xi_{2}=b_{1} \tilde{V}_{2}, \quad \xi_{1}=-\left(b_{2}+\tilde{V}_{0}\right) b_{1} q, \\
& \alpha_{1}=0, \quad \alpha_{2}=1, \quad \alpha_{3}=1, \quad \alpha_{4}=\frac{1}{2}, \quad \alpha_{5}=-\frac{1}{2}, \quad \alpha_{6}=\frac{1}{4}+\xi_{1}, \\
& \alpha_{7}=-\frac{1}{2}-\xi_{2}, \quad \alpha_{8}=\frac{1}{4}+\xi_{3}, \quad \alpha_{9}=\xi_{1}-\xi_{2}+\xi_{3}, \quad \alpha_{10}=1+2 \sqrt{\frac{1}{4}+\xi_{3}}, \\
& \alpha_{11}=2+2\left(\sqrt{\xi_{1}-\xi_{2}+\xi_{3}}+\sqrt{\frac{1}{4}+\xi_{3}}\right), \\
& \alpha_{13}=-\frac{1}{2}-\left(\sqrt{\xi_{1}-\xi_{2}+\xi_{3}}+\sqrt{\frac{1}{4}+\xi_{3}}\right) .
\end{aligned}
$$

We calculate the parameters required for the method

$$
\begin{aligned}
& \pi(s)=\frac{1-s}{2} \\
& \pm\left\{\left[\frac{1}{4}+\left(b_{1}\left(b_{2}+\tilde{V}_{0}+\tilde{V}_{1}\right)-k\right)\right] s^{2}+\left(-\frac{1}{2}-b_{1} \tilde{V}_{2}+k\right) s+\frac{1}{4}-b_{1}\left(b_{2}+\tilde{V}_{0}\right) q\right\}^{\frac{1}{2}},
\end{aligned}
$$

where

$$
\begin{aligned}
k_{1,2}=\left[b_{1}\left(\tilde{V}_{2}-\left(b_{2}+\tilde{V}_{0}\right) q\right)\right] \\
\quad \pm 2\left\{\left[\frac{1}{4}-b_{1}\left(b_{2}+\tilde{V}_{0}\right) q\right]\left[b_{1} b_{2}(1-q)+b_{1}\left(\tilde{V}_{0}(1-q)+\tilde{V}_{1}-\tilde{V}_{2}\right)\right]\right\}^{\frac{1}{2}} .
\end{aligned}
$$

Different $k$ 's lead to the different $\pi$ 's. For

$$
k=\left[b_{1}\left(\tilde{V}_{2}-\left(b_{2}+\tilde{V}_{0}\right) q\right)\right]-2\left\{\left[\frac{1}{4}-b_{1}\left(b_{2}+\tilde{V}_{0}\right) q\right]\left[b_{1} b_{2}(1-q)+b_{1}\left(\tilde{V}_{0}(1-q)+\tilde{V}_{1}-\tilde{V}_{2}\right)\right]\right\}^{\frac{1}{2}}
$$

$\pi(s)$ becomes

$$
\pi(s)=\frac{1-s}{2}
$$


$\left.-\left[\sqrt{\left[b_{1} b_{2}(1-q)+b_{1}\left(\tilde{V}_{0}(1-q)+\tilde{V}_{1}-\tilde{V}_{2}\right)\right.}\right]+\sqrt{\left[\frac{1}{4}-b_{1}\left(b_{2}+\tilde{V}_{0}\right) q\right]}\right] s+\sqrt{\left[\frac{1}{4}-b_{1}\left(b_{2}+\tilde{V}_{0}\right) q\right]}$,

and

$$
\begin{aligned}
& \tau(s)=1-2 s \\
& \left.-2\left[\sqrt{\left[b_{1} b_{2}(1-q)+b_{1}\left(\tilde{V}_{0}(1-q)+\tilde{V}_{1}-\tilde{V}_{2}\right)\right.}\right]+\sqrt{\left[\frac{1}{4}-b_{1}\left(b_{2}+\tilde{V}_{0}\right) q\right]}\right] s+\sqrt{\left[\frac{1}{4}-b_{1}\left(b_{2}+\tilde{V}_{0}\right) q\right]},
\end{aligned}
$$

where $\tau^{\prime}(s)<0$.

Therefore, we obtain the energy eigenvalue equation for the potential under the consideration [20, 45],

$$
\begin{aligned}
& \left.n^{2}+n\left(1+2 \sqrt{\left[b_{1} b_{2}(1-q)+b_{1}\left(\tilde{V}_{0}(1-q)+\tilde{V}_{1}-\tilde{V}_{2}\right)\right.}\right]+2 \sqrt{\left[\frac{1}{4}-b_{1}\left(b_{2}+\tilde{V}_{0}\right) q\right]}\right) \\
& +\left(\sqrt{\left[b_{1} b_{2}(1-q)+b_{1}\left(\tilde{V}_{0}(1-q)+\tilde{V}_{1}-\tilde{V}_{2}\right)\right]}+\sqrt{\left[\frac{1}{4}-b_{1}\left(b_{2}+\tilde{V}_{0}\right) q\right]}\right) \\
& \times\left(1+2 \sqrt{\left[\frac{1}{4}-b_{1}\left(b_{2}+\tilde{V}_{0}\right) q\right]}\right)-b_{1} \tilde{V}_{2}=0 .
\end{aligned}
$$

We write the corresponding unnormalized eigenfunctions are obtained in terms of the functions,

$$
\rho(s)=s^{2 \sqrt{\left[\frac{1}{4}-b_{1}\left(b_{2}+\tilde{V}_{0}\right) q\right]}}(1-s)^{2 \sqrt{\left[b_{1} b_{2}(1-q)+b_{1}\left(\tilde{V}_{0}(1-q)+\tilde{V}_{1}-\tilde{V}_{2}\right)\right]}},
$$

and

$$
\left.y_{n}(s)=P_{n}^{\left(2 \sqrt{\left[\frac{1}{4}-b_{1}\left(b_{2}+\tilde{V}_{0}\right) q\right]}\right.}, \quad 2 \sqrt{\left[b_{1} b_{2}(1-q)+b_{1}\left(\tilde{V}_{0}(1-q)+\tilde{V}_{1}-\tilde{V}_{2}\right)\right]}\right)(1-2 s),
$$

and

$$
\phi(s)=s^{\frac{1}{2}+\sqrt{\left[\frac{1}{4}-b_{1}\left(b_{2}+\tilde{V}_{0}\right) q\right]}}(1-s)^{\sqrt{\left[b_{1} b_{2}(1-q)+b_{1}\left(\tilde{V}_{0}(1-q)+\tilde{V}_{1}-\tilde{V}_{2}\right)\right]}},
$$

Now, let us give the corresponding upper Dirac spinor. Using [25], one obtains

$$
F_{n k}(s)=c_{n} s^{\left.\frac{1}{2}+\sqrt{\left[\frac{1}{4}-b_{1}\left(b_{2}+\tilde{V}_{0}\right) q\right.}\right]}(1-s)^{\sqrt{\left[b_{1} b_{2}(1-q)+b_{1}\left(\tilde{V}_{0}(1-q)+\tilde{V}_{1}-\tilde{V}_{2}\right)\right]}}
$$




$$
\times P_{n}^{\left.\left(2 \sqrt{\left[\frac{1}{4}-b_{1}\left(b_{2}+\tilde{V}_{0}\right) q\right]}, 2 \sqrt{\left[b_{1} b_{2}(1-q)+b_{1}\left(\tilde{V}_{0}(1-q)+\tilde{V}_{1}-\tilde{V}_{2}\right)\right.}\right)\right)}(1-2 s),
$$

where $C_{n}$ is a normalization constant and it is determined by the condition

$$
\int_{-\infty}^{\infty} F_{n k}(s) d s=1 .
$$

\subsection{Spin symmetry with a tensor coupling}

Substituting $q=1$ in (1), Eqs. (1) and (2) into (14) and considering spin symmetry (the condition of spin symmetry $d \Delta(r) / d r=0$ or $\Delta(r)=$ const $\left.=C_{s}\right)$ [47, 48], we obtain the second-order differential equation for the upper component of the Dirac spinor as follows,

$$
\begin{aligned}
& \left\{\frac{d^{2}}{d r^{2}}-\frac{(k+H)(k+H+1)}{r^{2}}-\left(M-E_{n k}\right)\left(E_{n k}+M-C_{s}\right)\right. \\
& \left.-\left(E_{n k}+M-C_{s}\right)\left(V_{0}+V_{1} \operatorname{coth}^{2} \alpha r+V_{2} \frac{\operatorname{coth} \alpha r}{\sinh \alpha r}\right)\right\} F_{n k}(r)=0 .
\end{aligned}
$$

By using the approximation scheme suggested by Greene and Aldrich [49]

$$
\frac{1}{r^{2}} \approx \frac{\alpha^{2}}{\sinh ^{2} \alpha r}
$$

and $s=\cosh \alpha r$, we have

$$
\begin{aligned}
& {\left[\frac{d^{2}}{d s^{2}}+\frac{-s}{1-s^{2}} \frac{d}{d s}\right.} \\
& \left.+\frac{1}{\left(s^{2}-1\right)^{2}}\left[-b_{1}\left(b_{2}+\tilde{V}_{0}+\tilde{V}_{1}\right) s^{2}-b_{1} \tilde{V}_{2} s+\left(-b_{3}+b_{1} b_{2}+b_{1} \tilde{V}_{0}\right)\right]\right] F_{n k}(s)=0,
\end{aligned}
$$

where

$$
b_{3}=(k+H)(k+H+1) \text {. }
$$

Following to the report given in the researcher [20,45], we obtain the Energy eigenvalue equation for the potential under the consideration

$$
\begin{aligned}
& n^{2}+n\left(1+2 \sqrt{b_{1}\left(\tilde{V}_{1}-\tilde{V}_{2}\right)+b_{3}}+2 \sqrt{\frac{1}{4}-\left(-b_{3}+b_{1} b_{2}+b_{1} \tilde{V}_{0}\right)}\right) \\
& +\left(1+2 \sqrt{\frac{1}{4}-\left(-b_{3}+b_{1} b_{2}+b_{1} \tilde{V}_{0}\right)}\right)\left(\sqrt{b_{1}\left(\tilde{V}_{1}-\tilde{V}_{2}\right)+b_{3}}+\sqrt{\frac{1}{4}-\left(-b_{3}+b_{1} b_{2}+b_{1} \tilde{V}_{0}\right)}\right)-b_{1} \tilde{V}_{2}=0
\end{aligned}
$$


Now, let us give the corresponding lower Dirac spinor. Using [25], one obtains

$$
\begin{aligned}
& F(s)=a_{n} s^{\frac{1}{2}+\sqrt{\frac{1}{4}-\left(-b_{3}+b_{1} b_{2}+b_{1} \tilde{V}_{0}\right)}}(1-s)^{\sqrt{b_{1}\left(\tilde{V}_{1}-\tilde{V}_{2}\right)+b_{3}}} \\
& \times P_{n}^{\left(2 \sqrt{\frac{1}{4}-\left(-b_{3}+b_{1} b_{2}+b_{1} \tilde{V}_{0}\right)}, 2 \sqrt{b_{1}\left(\tilde{V}_{1}-\tilde{V}_{2}\right)+b_{3}}\right)}(1-2 s) .
\end{aligned}
$$

where $a_{n}$ is a normalization constant and it is determined by the condition

$$
\begin{aligned}
\int_{-\infty}^{\infty} F_{n k}(s) d s & =1 \text {, the lower component of Dirac spinor can be calculated by using (12) as } \\
G_{n k}(r) & =\frac{1}{M+E_{n k}-\Delta(r)}\left(\frac{d}{d r}+\frac{k}{r}-U(r)\right) F_{n k}(r),
\end{aligned}
$$

\section{Conclusion}

We have investigated the Dirac equation with q-deformed hyperbolic Scarf potential for swave and including Coulomb-like tensor coupling in the case of spin symmetry. By using the approximation scheme and the parameteric generalization of the Nikiforov-Uvarov method, we have obtained the energy eigenvalues equation and the unnormalized wave functions.

\section{References}

1. W. Griner, Relativistic Quantum Mechanics-wave equation, $3^{\text {rd }}$ ed., (Springer-Verlag, Berlin, 2000).

2. J. D. Bjorken, S.D. Drell, Relativistic Quantum Mechanics (McGraw-Hill, NY, 1964).

3. J. N. Ginocchio, Phys. Rev. Lett. 78, 436 (1997). doi:10.1103/PhysRevLett.78.436

4. P. R. Page and T. Goldman, J. N. Ginocchio, Phys. Rev. Lett. 86, 204 (2001). doi:10.1103/PhysRevLett.86.204

5. A. Bohr, I. Hamamoto, and B. R. Mottelson, Phys. Scr. 26, 267 (1982). doi:10.1088/0031-8949/26/4/003

6. J. Dudek, W. Nazarewicz, Z. Szymanski, and G.A. Leander, Phys. Rev. Lett. 59, 1405 (1987). doi:10.1103/PhysRevLett.59.1405

7. A. Arima, M. Harvey, and K. Shimizu, Phys. Lett. B 30, 517 (1969). doi:10.1016/0370-2693(69)90443-2

8. K.T. Hecht and A. Adler, Nucl. Phys. A 137, 129 (1969). doi:10.1016/0375-9474(69)90077-3

9. O. Aydogdu and R. Sever, Ann. Phys. 325, 373 (2010). doi:10.1016/j.aop.2009.10.009

10. S. M. Ikhdair and R. Sever, Appl. Math. Com. 216, 545 (2010), arXiv:1001.3943v1 [math-ph] 22 Jan 2010.

11. J. Y. Guo and Z. Q. Sheng, Phys. Lett. A 338, 90 (2005). doi:10.1016/j.physleta.2005.02.026

12. L.H. Zhang, X. P. Li, and C. S. Jia, Phys. Lett. A 372, 2201 (2008). doi:10.1016/j.physleta.2007.11.022

13. C. S. Jia, T. Chen, and L. G. Gui, Phys. Lett. A 373, 1621 (2009). doi:10.1016/j.physleta.2009.03.006

14. Z. Fang, W. Yong, and G. Jian-You, Commun. Theor. Phys. (Bijing, China), 52, 813 (2009). 
doi:10.1088/0253-6102/52/5/09

15. M. R. Setare and S. Haidari, Int. J. Theor. Phys. 48, 3249 (2009). doi:10.1007/s10773-009-0128-5

16. G. Jian-You, M. Jie, and X. Fu-Xin, Chin. Phys. Lett. 20, 602 (2003). doi:10.1088/0256-307X/20/5/303

17. C. S. Jia, J. Y. Liu, P. Q. Wang, and X. Lin, Int. J. Theor. Phys. 48, 2633 (2009). doi:10.1007/s10773-009-0051-9

18. M. Hamzavi, A. A. Rajabi, and H. Hassanabadi, Phys. Lett. A 374, 4303 (2010). doi:10.1016/j.physleta.2010.08.065

19. H. Akcay and C. Tezcan, Int. J. Mod. Phys. C 20, 931 (2009). doi:10.1142/S0129183109014084

20. M. Hamzavi, H. Hassanabadi and A. A. Rajabi, Int. J. Theor. Phys. 50, 454 (2011). doi:10.1007/s10773-010-0552-6

21. S. Zarrinkamar, A. A. Rajabi and H. Hassanabadi, Ann. Phys. 325, 2522 (2010). doi:10.1016/j.aop.2010.05.013

22. H. Akcay, Phys. Lett. A 373, 616 (2009). doi:10.1016/j.physleta.2008.12.029

23. O. Audogdu and R. Sever, Few-Boby Syst. 47, 193 (2010). doi:10.1007/s00601-010-0085-9

24. M. Hamzavi, A. A. Rajabi and H. Hassanabadi, Few-Bady Syst. 48, 171 (2010). doi:10.1007/s00601-010-0095-7

25. S. M. Ikhdair and R. Sever, Appl. Math. Com. 216, 91 (2010).

26. C. Grosche, J. Phys. A: Math. Gen. 38, 2947 (2005). doi:10.1088/0305-4470/38/13/009

27. O. Yesiltas, Phys. Scr. 75, 41 (2007). doi:10.1088/0031-8949/75/1/006

28. D. Agboola. arXiv:1010.5981v1 [math-ph] 28 Oct 2010

29. A. Arda, R. Sever and C. Tezcan, Chin. Phys. Lett. 27, 010306 (2010). doi:10.1088/0256-307X/27/1/010306

30. S. M. Ikhdair and R. Sever. arXiv:0809.2485v1 [quant-ph] 15 Sep 2008

31. C. Berkdemir and J. Han, Chem. Phys. Lett. 409, 203 (2005). doi:10.1016/j.cplett.2005.05.021

32. A. N. Ikot, Appl. Phys. Res. 2, 202 (2010).

33. M. C. Zhang, G.H. Sun and S.H. Dong, Phys. Lett. A 374, 704 (2010). doi:10.1016/j.physleta.2009.11.072

34. S. Meyur and S. Debnath, Lat. Am. J. Phys. Educ. 4, 587 (2010).

35. H. Egrifes and R. Sever, Phys. Lett. A 344, 117 (2005). doi:10.1016/j.physleta.2005.06.061

36. A.D. Antia, A. N. Ikot and L.E. Akpabio, Euro. J. Scient. Res. 46, 107 (2010).

37. C. Berkdemir, A. Berkdemir and J. Han, Chem. Phys. Lett. A 417, 326 (2006). doi:10.1016/j.cplett.2005.10.039

38. H. Motavali, Mod. Phys. Lett. A 24, 1227 (2009). doi:10.1142/S0217732309028941

39. W. A. Yahya, K. J. Oyewumi, C. O. Akoshile and T. T. Ibrahim, J. Vect. Relat. 5 (3), 27 (2010).

40. A. Arda, R. Sever and C. Tezcan, Chinese J. Phys. 48, 27 (2010). Arxiv:0909.2086.

41. Y. F. Cheng and T. Q. Dai, Chin. J. Phys. 45, 480 (2007).

42. F. Zhou, Y. Wu and J. Y. Guo, Commun. Theor. Phys. (Beijibg, China) 52, 813 (2009).

43. Y. F. Cheng and T. Q. Dai, Commun. Theor. Phys. 48, 431 (2007). doi:10.1088/0253-6102/48/3/009

44. F. Yasuk, C. Berkdemir, and A. Berkdemir, J. Phys. A: Math. Gen. 38, 6579 (2005). doi:10.1088/0305-4470/38/29/012

45. C. Tezcan, R. Sever, Int. J. Theor. Phys. 48, 337 (2009). doi:10.1007/s10773-008-9806-y

46. A. Arai, J. Math. Anal. Appl. 158, 63 (1991). doi:10.1016/0022-247X(91)90267-4

47. J. Meng, K. Sugawara-Tanaha, S. Yamaji and A. Arima, Phys. Rev. C 59, 154 (1999). doi:10.1103/PhysRevC.59.154

48. J. Meng, K. Sugawara-Tanabe, S. Yamaji, P. Ring and A. Arima, Phys. Rev. C 58, R628 (1998). doi:10.1103/PhysRevC.58.R628

49. R .L. Greene and C. Aldrich, Phys. Rev. A 14, 2363 (1976). doi:10.1103/PhysRevA.14.2363 